\title{
Optimization and characterization of primaquine- loaded solid lipid nanoparticles (SLN) for liver schinonticide targeting by freeze drying
}

\begin{abstract}
The aim of this study was preparation of a liver schinonticide Primaquine phosphate (PQ) directly to the hepatocytes using solid lipid nanoparticles (SLN). The PQloaded solid lipid nanoparticles (PQ-SLNs) were prepared by a modified solvent emulsification evaporation method based on a water-in-oil-in-water $(w / o / w)$ double emulsion and dried freeze drying (PQ-SLNFD) to obtain the nanoparticles. The mean particle size, zeta potential, drug loading, and encapsulation efficiency of the PQSLNFD were $236 \mathrm{~nm},+23 \mathrm{mV}, 14 \%$, and $75 \%$, respectively. A spherical morphology of PQ-SLNFD was seen by scanning electron microscope had traces of drug crystals. In vitro, release profile depicted a steady drug release over 400 hours for PQ-SLNFD. Differential scanning calorimeter thermograms demonstrated presence of drug in drug-loaded nanoparticles along with disappearance of decomposition exotherms, suggesting increased physical stability of drug in prepared formulations. The nanoformulated PQ was $20 \%$ more effective as compared with conventional oral dose when tested in Plasmodium berghei-infected Swiss albino mice. This study established an effective process of developing a nanomedicine delivery system for PQ.
\end{abstract}

Keywords: double emulsion, solid lipid nanoparticles, freeze dried

\author{
Volume I Issue 3 - 2017
}

\author{
James Jorum Owuor, ${ }^{1,2}$ Florence Oloo, ${ }^{1,2}$ \\ Daniel Ouma,' Wesley Nyaigoti Omwoyo, ${ }^{2,4}$ \\ Jeremiah Waweru Gathirwa ${ }^{2,3}$ \\ 'Department of Chemical Sciences and Technology, Technical \\ University of Kenya, Kenya \\ ${ }^{2}$ Center for Research in Therapeutic Sciences, Strathmore \\ University Nairobi, Kenya \\ ${ }^{3}$ Kenya Medical Research Institute, Kenya; \\ ${ }^{4}$ Department of Chemistry, Maasai Mara University, Kenya
}

\begin{abstract}
Correspondence: James Jorum Owuor, Department of Chemical Sciences and Technology, Technical University of Kenya,
\end{abstract} Nairobi, Kenya, Email jjamesowuor@gmail.com

Received: November 13, 2017 | Published: December 14, 2017

\section{Introduction}

One of the strategies employed to improve PQ is reformulation into nanoparticles. Nanoparticles have been investigated for many applications due to the enhanced material properties that result from reduction in particle dimensions. The most active area of research is in the pharmaceutical industry, where nanoparticles have the potential to provide drug-delivery vehicles. ${ }^{1}$ Some advantages of nanoformulated drugs include maintaining drug therapeutic concentrations and circulatory time at target sites; protection from premature degradation in the gastrointestinal tract; improved pharmacokinetics, solubility, bioavailability, and stability; reduced toxicity; reduction in dose and dose frequency; enhancement of patient compliance; and prevention, reduction, or delay of onset of resistance. ${ }^{2}$

The main aim of using nanocarriers as drug-delivery systems is to promote drug protection against extracellular degradation, to improve selectivity in relation to the target, and to reduce dose frequency as well as duration of the treatment via enhancing the pharmacokinetic profile of the drug. ${ }^{3-5}$ Nanoformulation of drugs has made an impact in therapies for diseases like cancer. ${ }^{2}$ For example, Doxil ${ }^{\circledR}$ (Alza Corp., Mountain View, CA, USA) is a nanomedicine formulation of the anthracycline drug doxorubicin that is currently in use for cancer treatment. The four first-line antituberculosis drugs rifampicin, isoniazid, ethambutol, and pyrazinamide have been nanoformulated in polymeric nanoparticles. ${ }^{6,7}$ In contrast, nanotechnology has not been widely applied to transform therapies for poverty-related diseases such as malaria. Nevertheless, albumin and gelatin nanoparticles of PQ have been synthesized and shown to reduce toxicity in mice. ${ }^{8}$ Increased life-span index related to PQ-loaded nanoparticles was demonstrated after administration of free PQ diphosphate, free poly (diethyl methylidenemalonate) (poly [DEMM]) nanoparticles, and PQ-loaded poly (DEMM) nanoparticles as a single intraperitoneal injection to $P$. berghei-infected mice. ${ }^{9}$ In yet another study in mice, PQ-loaded liposomes were shown to protect nontarget tissues such as lung, kidneys, heart, and brain from PQ accumulation, thereby reducing the toxicity of $\mathrm{PQ} \cdot{ }^{10,11}$

The current study envisaged that reformulating PQ would enhance its efficacy and half-life, which may impact on its dosing regimen by enabling lower dosage and longer frequency. These will lead to reduced toxicity and better patient compliance. The strategy employed toward this was through the synthesis of PQ-loaded SLNs (PQ-SLNs). SLNs introduced in 1991 represent an alternative carrier system to traditional colloidal carriers such as emulsions, liposomes, and polymeric micro- and nanoparticles. ${ }^{12}$ SLNs are submicron colloidal carriers ranging from $50 \mathrm{~nm}$ to $1,000 \mathrm{~nm}$. The system consists of spherical solid lipid particles in the nanometer ranges, which are dispersed in water or in aqueous surfactant solution. The solid core contains the drug dissolved or dispersed in the solid high melting fat matrix..$^{13}$ The general ingredients include solid lipid(s), emulsifier(s), and water. A major advantage of SLNs is the fact that the lipid matrix is made from physiological lipids, which decreases the danger of acute and chronic toxicity. ${ }^{14}$ The solvent emulsification-evaporation method was selected for the formation of the nanoparticles. This method has emerged as a superior technique for preparing SLNs. A key step involves dispersions by precipitation in oil-in-water $(\mathrm{o} / \mathrm{w})$ emulsions. The lipophilic material is dissolved in a water-immiscible organic solvent that is emulsified in an aqueous phase. Upon evaporation of the solvent, nanoparticle dispersion is formed by precipitation of 
the lipid in the aqueous medium. ${ }^{15}$ Reproducibility of this method is reported to be high. ${ }^{16}$ The procedure used in forming the PQ-SLNs is outlined below.

The generation of SLN, the dispersion and control of the nanoparticles is a key stride. The attributes of the suspension determine the morphology of the particles and their properties. A few investigations have revealed the scattering and security of suspensions of nanosized SLN, for example, RAPAMUNE ${ }^{\circledR}$, EMEND ${ }^{\circledR}$, TriCor ${ }^{\circledR}$ and MEGACE $®$ among others as further explained in Table 1 .

Table I Current marketed pharmaceutical products utilizing nanocrystalline API

\begin{tabular}{|c|c|c|c|c|}
\hline Product & Drug compound & Indication & Company & Product \\
\hline RAPAMUNE® & Sirolimus & Immunosuppressant & Wyeth & Elan Drug Delivery Nanocrystals $\mathbb{R}$ \\
\hline EMEND® & Aprepitant & Antiemetic & Merck & Elan Drug Delivery Nanocrystals $\mathbb{R}$ \\
\hline TriCor ${ }^{\circledR}$ & Fenofibrate & $\begin{array}{l}\text { Treatment of } \\
\text { hypercholesterolemia }\end{array}$ & Abbott & Elan Drug Delivery Nanocrystals ${ }^{\circledR}$ \\
\hline MEGACE® & Megestrol acetate & Appetite stimulant & PAR Pharmaceutical & Elan Drug Delivery Nanocrystals ${ }^{\circledR}$ \\
\hline ES TriglideTM & Fenofibrate & $\begin{array}{l}\text { Treatment of } \\
\text { hypercholesterolemia }\end{array}$ & $\begin{array}{l}\text { First Horizon } \\
\text { Pharmaceutical }\end{array}$ & Skye Pharma IDD®-P technology \\
\hline
\end{tabular}

A strategy for the synthesis of nanoparticles is freeze drying. This strategy is getting extraordinary consideration these days for the synthesis of nanosized particles from inorganic salts and for the produce of porous bodies by a freeze casting process. The preparation of particles by this method was created with the target of maintaining a strategic distance of pressing aids to the particle surface Lyckfedt et al. ${ }^{6}$ However, it is extremely restricted when contrasted with spray drying. A principle highlight of freeze drying as a nanoformulation strategy is that the acquired nanoparticles have high porosity thus light granules can be produced Uchida et al., ${ }^{17}$ Yokota et al. ${ }^{18}$ The porosity and subsequently, the thickness of particles are controlled by the strong stacking of the suspensions, though the size distribution of the particles is a component of the viscosity and the solid substance of the suspension, the streaming rate utilized for spraying and the pressure of the connected gas Moritz et al. ${ }^{10}$ and Rundgren et al. ${ }^{19}$

The present research envisaged reformulating PQ to enhance its efficacy and half-life, which may impact on its dosing regimen by enabling lower dosage and longer frequency. These will lead to reduced toxicity and better patient compliance. The strategy employed toward this was through the synthesis of PQ-loaded Solid Lipid Nanoparticles (PQ-SLNs).

\section{Materials and methods}

\section{Materials}

All materials, reagents, chemicals, and PQ base utilized as a part of the study were provided by our partners at the Novartis, Basel, Switzerland. The stearic acid (SA), chitosan low-viscous, polyvinyl alcohol (PVA) of molecular weight 13,000-23,000 and partially hydrolyzed (87\%-89\%), D-lactose monohydrate, sulfanoyl, and ethyl acetate (EtOAc) were obtained from Sigma-Aldrich (Basel, Switzerland), and Pluronic ${ }^{\circledR}$ F127 Prill from BASF Corporation (Mount Olive, NJ, USA). All other synthetic items were commercially accessible and of analytical grade. In this test outline, stearic acid was the lattice; PVA and pluronic were surfactants stabilised the emulsion, and chitosan was a mucoadhesive expanding circulation time in the digestive system to enable the majority of the nanoparticles to be assimilated. Lactose improved particle size reduction, since it is a binder, while sulfanoyl was used as an antifoaming agent.

\section{Preparation of SLNs via freeze drying}

The nanoparticles loaded with PQ were prepared using a modified multiple emulsion solvent evaporation technique followed by freeze drying. A solution of $100 \mathrm{mg}$ PQ was dissolved in $2 \mathrm{~m}$ of aqueous $2 \%$ PVA to form the aqueous phase containing the drug. The organic phase was made by dissolving $50 \mathrm{mg}$ of stearic acid in $10 \mathrm{ml}$ of EtOAC. The aqueous phase was often dispersed in the organic phase by means of a high speed homogenizer (silverson L4R; Silverson machines limited, Buckinghamshire, UK), with a speed varying between 3,000 and 6,000rpm for 3 minutes. This water-in-oil (w/o) emulsion was transferred to a specific volume of an aqueous $2 \% \mathrm{w} / \mathrm{v}$ PVA (mw $=13000-23000), 5 \%$ of $0.2 \%(\mathrm{w} / \mathrm{v})$ chitosan low viscous, and $5 \mathrm{ml}$ of $5 \%(\mathrm{w} / \mathrm{v})$ D-lactose monohydrate. A drop of sulfanyl was added as a stabiliser to the resultant water-in-oil-in-water $(\mathrm{w} / \mathrm{o} / \mathrm{w})$ emulsion and the mixture was further emulsified by homogenisation at $8,000 \mathrm{rpm}$. The water-in-oil-in-water double emulsion obtained was gently stirred overnight at room temperature to remove the organic solvent. Thereafter, drug-loaded SLNs were harvested by ultracentrifugation (37000g for $15 \mathrm{mins}$ ) followed by a series of wash steps with deionized water. The recovered pellets were dispersed in liquid Nitrogen before freeze drying (Heto DRYWINNER, Germany) at $0.05 \mathrm{mBar}$ for 24 hours.

The nanoparticles loaded with PQ were synthesised utilizing a modified multiple emulsion solvent evaporation method followed after by freeze drying. A solution of $100 \mathrm{mg}$ PQ was dissolved in $2 \mathrm{~m}$ of fluid $2 \%$ PVA to shape the aqueous phase containing the drug. The organic stage was made by dissolving $50 \mathrm{mg}$ of stearic acid in $10 \mathrm{ml}$ of EtOAC. The aqueous stage was frequently dispersed in the organic stage by a technique of fast homogenizer (silverson L4R; Silverson machines restricted, Buckinghamshire, UK), with a speed shifting in the vicinity of 3,000 and 6,000 rpm for 3 minutes. This water-in-oil (w/o) emulsion was transferred to a specific volume of 
an aqueous $2 \% \mathrm{w} / \mathrm{v}$ PVA (mw=13000-23000), $5 \%$ of $0.2 \%(\mathrm{w} / \mathrm{v})$ chitosan low thick, and $5 \mathrm{ml}$ of $5 \%(\mathrm{w} / \mathrm{v}) \mathrm{D}$-lactose monohydrate. A drop of sulfanyl was added as a stabilizer to the resultant water-in-oilin-water (w/o/w) emulsion and the blend was additionally emulsified by homogenisation at $8,000 \mathrm{rpm}$. The water-in-oil-in-water twofold emulsion got was delicately blended overnight at room temperature to expel the organic solvents. Drug loaded SLNs were collected by ultracentrifugation ( $37000 \mathrm{~g}$ for $15 \mathrm{mins}$ ) followed by a progression of wash steps with deionized water. The recovered pellets were scattered in fluid Nitrogen before freeze drying (Heto DRYWINNER, Germany) at $0.05 \mathrm{mBar}$ for 24 hours.

\section{Particle characterisation}

Determination of size and $\zeta$-potential: A Zetasizer Nanoseries (Malvern Instruments, Malvern, UK) was utilized to estimate particle size, poly dispersity index(PDI) and $\zeta$-potential of all the two sorts of particles by dynamic light scattering (DLS) or photon correlation rule. All scattering-samples were diluted 1:500 in ultra-pure water, vortex and sonicated for determination of the, PDI and $\zeta$-potential Three replicates of each were measured at room temperature. Results are presented as mean \pm standard deviation.

Stability study and $\mathbf{p H}$ determination: To guarantee stability of the formulations over some stretch of time, scatterings in water $(0.5 \mathrm{mg} /$ $\mathrm{ml}$ ) were checked for up to 2 months after processing of SLNwith respect to particle size, PDI, $\zeta$-potential, and $\mathrm{pH}$. For the $\mathrm{pH}$ deduction acalibrated potentiometer (Model AZ-8306: AZ Instruments Corp., Taichung City, Taiwan) was utilized. $\mathrm{pH}$ values were measured by the direct immersion of the electrode in the undiluted dispersion at $10 \%$ $(\mathrm{w} / \mathrm{v})$. The samples were stored in amber glass flasks at $25^{\circ} \mathrm{C}$.

\section{Drug loading and encapsulation efficiency}

Drug content was broke analyzed after a modified form of the technique outlined by Fontana et al., ${ }^{20} \mathrm{EE} \%$ was resolved utilizing both the direct and indirect strategy. In the indirect strategy, $20 \mathrm{mg}$ of the synthesised nanoparticles were scattered in $10 \mathrm{~mL}$ of water and vortexed in falcon tubes until completely dispersed. The resultant solution was then ultra-centrifuged at $15,000 \mathrm{rpm}$ for 20 minutes at $4^{\circ} \mathrm{C}$. The supernatant was then taken for ultraviolet-visible (UVVIS) investigation at wavelengths between $400 \mathrm{~nm}$ and $200 \mathrm{~nm}$. For the direct technique, the precipitate was taken and disintegrated in a predetermined amount of EtOAc. Water was then added to the solution and left overnight. The aqueous stage was then isolated by means of aseparating funnel and examined for PQ concentration. The concentration of the drug was calculated by methods for a standard curve as separated from UV-VIS spectrometry examination by utilizing diverse known concentrations of the drug. The EE\% and DL\% were ascertained utilizing the equations underneath. ${ }^{21-24}$

$\mathrm{EE} \%=($ drug in precipitate/total added drug $) \times 100(1)$

$\mathrm{DL} \%=($ drug in precipitate $/$ drug in precipitate + added excipients $) \times 100(2)$

Where "drug in precipitate"=total drug added -free drug after ultra-centrifugation (indirect method) and "added excipients"=lipids+surfactant mixtures + other ingredients used.

\section{Surface morphology}

Scanning electron microscopy was utilized to give an approach to specifically observe the morphological appearance of the nanoparticles.
The particles were first covered in gold to limit the impact of heat amid high-power amplification and were then run through a scanning electron microscope instrument (SU1510 model; Hitachi Ltd., Tokyo, Japan) where photos of the nanoparticles were taken. Fourier change infrared spectroscopy (FTIR) was likewise used to decide the useful gatherings exhibit on the surface of the nanoparticle.

\section{In vitro release experiment}

In vitro release studies were performed utilizing the strategy outlined by Mühlen and Mehnert, with slight modification. To decide if the encapsulation procedure brings about a sustained release of drug was performed in phosphate buffered saline (0.1M PBS, $\mathrm{pH}$ 7.4). in this strategy, three replicates of each sample containing nanoparticles of a known concentration of PQ-stacked Freeze Drying (FD) nanoparticles strategies were set in a dialysis film and immersing dialysis film into in $20 \mathrm{ml}$ of PBS arrangement in a shaker at $37^{\circ} \mathrm{C}$, at a predetermined interim $100 \mu \mathrm{L}$ of sample were analysed by HPLC utilizing the technique adapted from Mohan, with minor alterations. For positive controls, $10 \mathrm{mg}$ of free PQ drug was dialysed into $20 \mathrm{ml}$ of PBS sample. The investigation gave the amount of drug discharged from the nanoparticles with time..$^{25-30}$

\section{In vitro antimalarial assays}

In vitro cultivation of malaria parasite: The asexual intraerythrocytic stage of P. falciparum laboratory strain (3D7) was obtained from the Centre of Traditional Medicine and Drug Research (CTMDR), Kenya Medical Research Institute (KEMRI) The parasites were continuously cultured in vitro according to the method of Hout (2006) with slight modifications.

Frozen parasite vials stored in liquid nitrogen were thawed in water bath at $37^{\circ} \mathrm{C}$ for $15 \mathrm{~min}$, spun at $2000 \mathrm{rpm}$ for $10 \mathrm{~min}$ and the supernatant discarded. The pellets were transferred into a sterile $15 \mathrm{~mL}$ falcon tube and equal volume of thawing mix $(3.5 \% \mathrm{NaCl}$ in distilled water) was added, thoroughly mixed and spun at 2000rpm for $10 \mathrm{~min}$. The supernatant was removed and $1 \mathrm{~mL}$ of CPM (complete parasite medium) was added and spun again for $2000 \mathrm{rpm}$ for $10 \mathrm{~min}$. The washing step was repeated and supernatant discarded. The complete parasite medium ( $\mathrm{pH}$ 7.3) used consisted of filter-sterilized RPMI 1640 solution supplemented with $0.5 \%$ AlbuMAX II, hypoxanthine $(0.04 \%)$, buffered with $0.4 \%$ sodium bicarbonate $\left(\mathrm{NaHCO}_{3}\right), 0.72 \%$ $\mathrm{N}$-2-hydroxyethylpiperazine-N-2-ethanesulfonic acid (HEPES) and $0.005 \mathrm{mg} / \mathrm{mL}$ gentamicin. The pellets were suspended in $25 \mathrm{~mL}$ culture flask (BD falcon) containing $5 \mathrm{~mL}$ of CPM and $200 \mu \mathrm{L}$ freshly prepared packed $\mathrm{RBC}$ (sickling negative, $\mathrm{O}$ rhesus positive) to have a haematocrit of $4 \%$.

The culture was flushed with a mixture of gases (2\% Oxygen, $5.5 \%$ Carbon dioxide and $92.5 \%$ Nitrogen) for 30s. The flask was closed and placed in an incubator (RS Biotech, Livingston, UK) set at $37^{\circ} \mathrm{C}$. The culture medium was changed daily using fresh CPM. The parasitaemia was checked by preparing a thin smear on a microscope slide under sterile conditions in the laminar flow safety cabinet (Hitachi Clean Bench, Japan). The slides after drying were fixed in absolute methanol, stained with $10 \%$ Giemsa in phosphate buffer for $10 \mathrm{~min}$. The slides were examined under the light microscope using $\times 100$ (oil immersion) objective lens for level of parasitaemia, growth stages and viability. The culture flask was re-incubatored after gassing and adding appropriate volume of CPM. The number of infected RBCs and total number of RBCs in a field were counted and 
recorded. This was done for three fields making up to at least a total of 300 RBCs. The percentage parasitaemia in each field was calculated using the formula:

$$
\% \text { Parasitaemia }=\frac{\text { Number of infected } R B C s}{\text { Total } R B C \text { s conted }} \times 100
$$

Parasitaemia levels above $4 \%$ were harvested and used for assays or diluted with appropriate volume of packed RBCs to $1 \%$ parasitaemia. The parasites were maintained in continuous culture to obtain a stable parasitaemia before they were used for the efficacy assay. ${ }^{31-33}$

\section{In vitro drug sensitivity assay}

The in vitro antimalarial activity of nanoformulated primaquine and in combination with standard antimalarial agents against $P$. falciparum (3D7, Chloroquine sensitive) was investigated using the SYBR Green I-based fluorescence assay. Cultures were synchronized by two rounds of sorbitol $(5 \%)$ treatment to obtain a highly synchronous ring stage parasites used in each assay. Stock drug solutions were prepared in $70 \%$ ethanol except for chloroquine which was dissolved in distilled water. Nanoformulated primaquine (NP), chloroquine (CQ) and Free Primaquine (PQ) stocks were at $0.5 \mathrm{mM}$. Concentrations ranging from 32.5 to $2080 \mathrm{nM}$ for NP, 2 to $640 \mathrm{nM}$ for PQ and 3.2 to $83 \mathrm{nM}$ for CQ were used for the interaction assays. Nanoformulated primaquine plus primaquine $(\mathrm{PQ})$ solutions were prepared in assay medium at ratios of $5: 0,4: 1,3: 2,2: 3,1: 4$ and $0: 5$ followed by 2 -fold serial dilutions in assay medium of each ratio, allowing the $\mathrm{IC}_{50}$ to fall approximately at the mid-point of the serial dilution of each drug alone (Fivelman 2004). For each combination assay, $90 \mu \mathrm{L}$ of parasite culture were seeded into each culture well, and $10 \mu \mathrm{L}$ of diluted drugs in complete medium solutions were dispensed into each well to obtain seven desired final concentrations. The haematocrit of cultures were adjusted to $2 \%$ by dilution with complete parasite medium and parasitaemia was stepped down to $1 \%$ with washed uninfected RBCs. The plates were arranged in a clean modular incubation chamber (Billups-Rothenberg Inc, USA) and flushed with mixed gas for 6 min. The chamber with the assay plates was placed in an incubator for $48 \mathrm{~h}$ and set at $37^{\circ} \mathrm{C}$. After the 48-h incubation, the plates were wrapped in aluminium foil and stored at $-30^{\circ} \mathrm{C}$ overnight.

The plates were thawed and mixed with $100 \mu$ of $S Y B R{ }^{\circledR}$ Green lysis buffer $(0.008 \% \mathrm{w} / \mathrm{v}$ Saponin, $0.08 \% \mathrm{w} / \mathrm{v}$ Triton X-100, $5 \mathrm{mM}$ EDTA and 20mM Tris base). The plates were incubated in the dark at room temperature for about $1 \mathrm{~h}$ and fluorescence data were acquired using fluorescence multi-well plate reader (Tecan Infinite M200 Pro) with excitation and emission wavelength at 485 and $535 \mathrm{~nm}$, respectively. The experiment was done in triplicate (Table 2). ${ }^{34-38}$

\section{In vivo antimalarial assay}

Animals: Mice infected with chloroquine-sensitive strain of Plasmodium berghei NK-65 were obtained from the Animal foundry of the Centre of Traditional Medicine and Drug Research (CTMDR), Kenya Medical Research Institute (KEMRI). The parasites were kept alive by continuous intraperitoneal passage in mice every 4 days (Adzu and Haruna 2007). These infected mice were used for the study. Prior to the study, one of the infected mice was kept and observed to produce disease symptoms similar to human infection (English, 1996). The use of animals was in agreement with the National Institute of Health Guidelines for Care and use of laboratory animals (1985) and were approved by the Ethical Review Committee of the Kenya Medical Research Institute (KEMRI)

\section{Antimalarial assay of nanoformulated primaquine hydrochloride}

The $\mathrm{ED}_{50}$ of nanoformulated primaquine was confirmed using the curative (Rane) test as described by Ryley and Peters (1970). In this test schizonticidal activity of nanoformulated primaquine was determined in established infection. On day 0 of this test, the percentage parasitaemia and red blood count of the donor mice were determined by using a Giemsa-stained thin blood smear of the donor mice and improved Neubaur Counting Chamber, respectively. Blood of the donor mice was collected by cardiac puncture and diluted with physiological saline to give a concentration of $1 \times 10^{6}$ parasitized erythrocytes per $\mathrm{mL}$. The mice were injected intraperitoneally with $0.2 \mathrm{ml}$ of standard inoculum of $1 \times 10^{6} P$. berghei NK 65 infected erythrocytes on the first day (day 0 ). After $72 \mathrm{~h}$, and following confirmation of parasitaemia, the mice were divided into six groups of five mice per group. These groups were treated with nanoformulated primaquine at doses of $3,10,30$, and $100 \mathrm{mg} / \mathrm{kg}$ p.o. The positive control group was treated with Chloroquine $(4 \mathrm{mg} / \mathrm{kg})$ and an equal volume of physiological saline was given to the negative control group. The treatment lasted for 6 days at a single dose per day after which blood smears were collected and examined microscopically to monitor the parasitaemia level.

\section{In vivo antimalarial interaction assay}

The curative or Rane test was used to evaluate the antimalarial properties of the combination of nanoformulated primaquine (NP) $(40 \mathrm{mg} / \mathrm{kg})$ and free primaquine $(\mathrm{PQ})\left(\mathrm{ED}_{50}=6 \mathrm{mg} / \mathrm{kg}\right)$. The mice were injected intraperitoneally with standard inoculum of $1 \times 10^{6} \mathrm{P}$. berghei NK 65 infected erythrocytes on the first day (day 0). After $72 \mathrm{~h}$, and following confirmation of parasitaemia, the mice were divided into seven groups of five mice per group. The first two groups were treated with nanoformulated primaquine (NP) $(40 \mathrm{mg} / \mathrm{kg})$ and primaquine $(6 \mathrm{mg} / \mathrm{kg})$, respectively. The doses selected were based on the $\mathrm{ED}_{50}$ of nanoformulated primaquine obtained from previous experiment as well as the reported $\mathrm{ED}_{50}$ of primaquine against $P$. berghei-infected mice (Vivas 2007). Four combinations of the two antimalarial agents of varying concentrations were used. Nanoformulated primaquine: Primaquine (40mg/kg: $6 \mathrm{mg} / \mathrm{kg}), \quad(20 \mathrm{mg} / \mathrm{kg}$ : $3 \mathrm{mg} / \mathrm{kg}), \quad(10 \mathrm{mg} / \mathrm{kg}$ : $1.5 \mathrm{mg} / \mathrm{kg}),(5 \mathrm{mg} / \mathrm{kg}: 0.75 \mathrm{mg} / \mathrm{kg})$. This was in combinations of $1: 1$, $1 / 2: 1 / 2,1 / 4: 1 / 4$, and $1 / 8: 1 / 8$ respectively, of a fixed dose ratio of $1: 1$. The negative control received equal amount of physiological saline daily for a period of six days just as the drug-treated groups. On day 3 of inoculation, parasitaemia was confirmed and treatment with the drug combinations began. Thin blood smears of the test mice stained with $10 \%$ Giemsa solution were used to determine the percentage parasitaemia microscopically, by counting 4 fields of approximately 100 erythrocytes per field.

\section{Statistical analysis of data}

In vitro interaction assay: $\mathrm{IC}_{50}$ value (drug concentration that inhibits the parasite growth by $50 \%$ ) was used as an indicator of antimalarial activity and was determined from log dose-response curves plotted using GraphPad Prism (GraphPad 6 Software, San Diego). The $\mathrm{IC}_{50}$ values were determined by analysis of dose-response curves. Growth inhibition due to nanoformulated primaquine and the other antimalarial 
agents defined as the difference between the percentage parasitaemia of each treatment group and the corresponding control was calculated. $\mathrm{IC}_{50}$ values were used to calculate the fractional $\mathrm{IC}_{50} \mathrm{~s}\left(\mathrm{FIC}_{50} \mathrm{~s}\right)$ for each drug ratio. $\Sigma \mathrm{FIC}_{50} \mathrm{~s}$ of nanoformulated primaquine with the standard antimalarials were calculated by the following equation:

$$
\text { ÓFIC } 50=\left(\frac{I C_{50} \text { of Nanoformulated } \mathrm{PQ} \text { in combination }}{I C_{50} \text { of Nanoformulated } \mathrm{PQ} \text { alone }}\right)+\left(\frac{I C_{50} \text { of } \mathrm{PQ} \text { in combination }}{I C_{50} \text { of } \mathrm{PQ} \text { alone }}\right)
$$

$\Sigma \mathrm{FIC}_{50}$ values indicate the nature of the interactions as follows: $\Sigma \mathrm{FIC}_{50}<0.8$ is synergism; $\Sigma \mathrm{FIC}_{50} 0.8$ to 1.4 is additive, $\Sigma \mathrm{FIC}_{50}>1.4$ is antagonism. Mean $\Sigma \mathrm{FIC}_{50} \mathrm{~s}$ were used to classify the overall nature of the interaction (Snyder et al., 2007).

\section{In vivo interaction assay}

The antimalarial activity was determined by using the equation:

$$
\% \text { Suppresion }=\left[\frac{\text { Parasitaemia of }- \text { ve control }- \text { Parasitaemia of test }}{\text { Parasitaemia of }- \text { vecontrol }}\right] \times 100
$$

The potency of nanoformulated primaquine and primaquine were estimated from their log-dose response curves. The negative control received physiological saline for the duration of treatment. The estimated potencies $\left(\mathrm{ED}_{50}\right.$ 's) of nanoformulated primaquine and primaquine in both tests were also used to compute the theoretical potency (Zadd) as follows;

Zadd $=f\left(\mathrm{ED}_{50}\right)$ of primaquine $+(1-f) \mathrm{ED}_{50}$ of nanoformulated primaquine

Where $f$ is the fraction of each component in the mixture.

To obtain the combination potency of the co-administered drug candidates, the two agents were orally administered to mice three days post-intraperitoneal inoculation with $1 \times 10^{6} \mathrm{P}$. berghei NK 65 infected erythrocytes daily at doses of their respective $\mathrm{ED}_{50}$ 's and in fixed ratio combinations of fractions of their respective $\mathrm{ED}_{50}$ values of $1 / 2,1 / 4$, $1 / 8$. The treatment regime continued for 6 days. The experimental potency (Zexp) of the co-administered test agents was determined by least square method of regression. Data for toxicity studies were presented as Mean \pm S.E.M. The presence of significance differences among means of groups was determined by one-way ANOVA using GraphPad Prism for Windows version 5.0 (GraphPad Software, San Diego, CA, USA). Significance difference between pairs of groups was calculated using the Newman-Keul's Multiple Comparison Test.

\section{Results and discussion}

\section{Particle characterisation}

Particle size, size distribution and zeta potential: The measure of nanoparticles was optimized against a benchmark of $250 \mathrm{~nm}$ as it is accounted for smaller particles (normal size $<250 \mathrm{~nm}$ ) undergo passive diffusion through hepatocytes. Different parameters were optimized to acquire an average particle size ranging between 230 and $250 \mathrm{~nm}$ and an average polydispersity index of 0.1 to 0.2 .. The expansion of shear rate by expanding the speed of homogenizer caused a lessening in particle size.

All particles demonstrated a positive zeta potential. The added drops of chitosan to give positive surface charge brought about microparticles. The sizes measured for the two unique sorts of particles went between $\sim 250-260 \mathrm{~nm}$ (Table 1). FD gave rise to a generally larger size when contrasted to SD. This can be explained by the presence of the oil phase (U.M.A., 2009) prompting a coreand-shell structure for FD rather than a matrix system in the spray dried (SD). All nanoparticles had a PDI in the range of 23.48 and 15.50 affirming a homogeneous size appropriation. The $\zeta$-Potential of every one of the two sorts of NPs was positive, extending from 17.0 to $19.5 \mathrm{mV}$. This positive potential is in all likelihood caused by the polymeric divider made by the lipids. The strength and stability of the particles is because of steric obstruction of the surfactant between the two phases counteracting mixture preventing coalescence (Table 3 ).

\section{Stability study}

Stability of the NPs in suspension was tested for a period of 3 months in terms of size, polydispersity, $\zeta$-potential, and $\mathrm{pH}$ (Table 2). After this period a slight incrementin particle size was noted, probably as a resultof aggregation or agglomeration. However, this effect was minimal and negligible. The $\zeta$-potential varied marginally over time with no general trend observed. The $\mathrm{pH}$ study demonstrated that the $\mathrm{pH}$ values obtained were suitable for application on the skin over the entire period of three months, as the $\mathrm{pH}$ stayed between $\mathrm{pH}$ 5.1-6.1. A slight reduction in $\mathrm{pH}$ was observed, but still remained adequate for topical application. This effect has previously been explained as a possible degradation of the polymer due to a release of free PQ, as well as a hydrolysis of the medium chain triglycerides, leading to an increase of free fatty acids (Abdelwahed et al, 2006). Thus, all measurements confirmed that no noteworthy changes in size, polydispersity, $\zeta$-potential, or $\mathrm{pH}$ occurred over time (Table 4).

\section{Drug loading and encapsulation efficiency}

The PQ content measured approximately $0.5 \mathrm{mg} / \mathrm{ml}$ for all two nano carriers, showing that 40 to $30 \%$ drug were lost during the preparation step for FD. Moreover, the encapsulation efficiency (EE \%) measured was close to 60 to $78 \%$, regardless of the type of particle (for exact values please refer to Table 1). Hence, no significant differences regarding the ability to encapsulate the drug were observed on FD although the preparation processes and components used were slightly different.

\section{Surface morphology}

Scanning electron microscopy (SEM) analysis was feasible for all two types of particles (Figure 1).

FD the imaging was more difficult, most likely due to the presence of the organogel. Both methods confirmed the expected morphology. All particles were spherical in shape, revealed a smooth surface, and a homogeneous size distribution (Figure 2).

The FTIR chromatogram in Figure 2 shows peaks of the drug formulations. The level of drug enclosure in the nanoparticles or absence in the case of the drug-free nanoparticles could be deduced from the chromatograms. Of particular interest was the $\mathrm{NH}_{2}$ bending at $1,614 \mathrm{~cm}^{-1}$ and aromatic $\mathrm{C}=\mathrm{C}$ stretching at around $1,532 \mathrm{~cm}^{-1}$ and $1,467 \mathrm{~cm}^{-1}$. As seen in Figure 4, free drug had more pronounced peaks in those particular absorbance regions, but in the case of the nanoparticles with $5 \mathrm{~mL}$ of lactose, the peaks appeared reduced. When $15 \mathrm{~mL}$ of lactose was used as a structural binder, the peaks reduced much further, indicating successful encapsulation in the solid lipid 
matrix as has been similarly observed in other studies.

\section{DTA}

DTA analysis gave the degradation points of the tested samples shown in Figure 3. The first depression in the curves was a measure of moisture content. From TGA analysis, sample moisture content was found to be $4 \%-5 \%$. A moisture content of less than $5 \%$ is acceptable in the pharmaceutical.

\section{In vitro release studies}

To determine whether the encapsulation process results in a sustained release of drug, the dialyzer dialysis membrane method was applied. The release profiles of the SLNs in suspension versus free drug in a PBS solution as a control is depicted in Figure 4. A noteworthy issue amid the work with lipid nano pellets has been the burst discharge seen with these systems. At the point when not washed properly, PQ-SLNs could demonstrate a burst discharge due to unencapsulated drug. An extended drug discharge has been acquired when contemplating the consolidation of prednisolone. As can obviously be found in Figure 4,no significant differences were observed between the release profiles of the two nano carriers $\mathrm{FD}(\mathrm{p}>0.05)$. However, as was anticipated, all the two PQ-loaded particles showed ansustaineddischarge of the drug, as following 24 hours just around half of the typified drug was discharged for each of the three carriers. Indeed, even toward the finish of a $72 \mathrm{~h}$ period, the mean concentration of discharged drug still stayed beneath $80 \%$. Free drug, conversely, showed a quick discharge profile with $\sim 80 \%$ of PQ as of now being distinguished after just $5 \mathrm{~h}$.

\section{In vivo antimalarial assay}

Determination of $\mathrm{ED}_{50}$ of Nanoformulated primaquine in $P$ berghei infected mice. Free Primaquine, Nanoformulated primaquine and standard antimalarial chloroquine produced a dose dependent reduction in parasitaemia levels with similar reduction as in the standard antimalarial chloroquine -treated group (positive control). The $\mathrm{ED}_{50}$ of Nanoformulated primaquineon the $5^{\text {th }}$ and $6^{\text {th }}$ days of treatment was calculated to be $10.45 \pm 0.5 \mathrm{mg} / \mathrm{kg}$ and $45 \mathrm{mg} / \mathrm{kg}$, respectively.
Suppressive efficacy against the malaria parasite was evaluated by comparing the percentage reduction of parasitemia (chemosuppression) between the treated and untreated groups. Results are shown in Table 5. Average parasitemia in both nanoformulated and free PQ drug were significantly lower $(P<0.05)$ than that of the untreated group for both test concentrations. There was no significant difference in parasitemia levels and survival time between the unloaded nanoparticles and the untreated control group, indicating that the empty nanoparticles and the excipients therein did not exert any antimalarial activity. When mice were treated with PQ-SLNs at a dose of $2 \mathrm{mg} / \mathrm{kg} / \mathrm{day}$, chemosuppression of $93.5 \%$ was observed. In comparison, only $71.9 \%$ chemosuppression was observed when mice were treated with free PQ at a similar dose. This indicated that nanoformulation of PQ increased its efficacy by more than $20 \%$. The mean survival time of mice treated with the nanoformulated PQ was similarly enhanced when compared with the group of mice that received the conventional dose of $\mathrm{PQ}$. In a previous study, it was observed that formulating PQ into the nanoemulsion shows effective antimalarial activity against $P$. berghei infection in Swiss albino mice at $25 \%$ lower dose level compared with the conventional oral dose. 11

\section{Combination antimalarial assay}

Table 5 shows the antimalarial activity, thus the mean percentage reduction in parasitaemia produced by the drugs alone or in nanoformulated form, compared to the control, on days 1 to 6 . The Nanoformulated primaquine $\left(\mathrm{ED}_{50}=40 \mathrm{mg} / \mathrm{kg}\right)$ and Nanoformulated primaquine $\left(\mathrm{ED}_{50}=6 \mathrm{mg} / \mathrm{kg}\right)$ produced a significant reduction in parasitaemia from days 1 and 6 . The Nanoformulated primaquine at all dose levels produced high percentage suppression in the first three days compared to free primaquine only. The lowest dose ratio combination (1/8:1/8) showed high parasite levels on days 5 and 6 compared to the negative control group.

\section{In vivo synergistic interaction of nanoformulated primaquine and free primaquine}

The theoretical $\mathrm{ED}_{50} \mathrm{~s}$ of Free Primaquine and Nanoformulated primaquine combination was $8.3 \pm 0.35 \mathrm{mg} / \mathrm{kg}$. The experimental $\mathrm{ED}_{50}$ (Zexp) of the mixture was $1.03 \pm 0.03 \mathrm{mg} / \mathrm{kg}$. The degree of interaction calculated as the interaction index was 0.14 (Table 5).

Table 2 Combination ratio of nanoformulated primaquine to standard antimalarial drugs (PQ)

\begin{tabular}{|c|c|c|c|c|}
\hline \multirow{2}{*}{ Combination solution } & \multicolumn{2}{|c|}{ Ratio of nanoformulated primaquine to $P Q$} & \multicolumn{2}{|l|}{ Volume $(\mu \mathrm{L})$} \\
\hline & Nanoformulated PQ & Free PQ & Nanoformulated PQ & Free PQ \\
\hline 1 & 5 & 0 & 10 & 0 \\
\hline 2 & 4 & 1 & 8 & 2 \\
\hline 3 & 3 & 2 & 6 & 4 \\
\hline 4 & 2 & 3 & 4 & 6 \\
\hline 5 & 1 & 4 & 2 & 8 \\
\hline 6 & 0 & 5 & 0 & 10 \\
\hline
\end{tabular}


Table 3 Size distribution, PDI values, and $\zeta$-potential of nanoparticles (NP) using dynamic light scattering (DLS) analysis; mean \pm standard deviation

\begin{tabular}{llllll}
\hline Type of particle & Size $(\mathbf{n m})$ & PDI & $\zeta$-potential $(\mathbf{m V})$ & PQ DL(\%) & Encapsulation efficiency $(\%)$ \\
\hline FD & $267.6 \pm 3.7$ & $0.13 \pm 0.02$ & $17.17 \pm 1.11$ & $23.48 \pm 0.02$ & $78.46 \pm 1.0$ \\
& $228 \pm 2$ & $0.17 \pm 0.02$ & $13.59 \pm 0.73$ & - & -
\end{tabular}

Table 4 Physiochemical characteristics of Freeze Dried (FD and Spray Dried (SD) tracked for 2 months; mean \pm SD (size measured using DLS analysis)

\begin{tabular}{llllll}
\hline Type of particle & Month & Size $(\mathbf{n m})$ & PDI & $\zeta$-Pot $(\mathbf{m V})$ & pH \\
\hline & 0 & $295 \pm 4$ & $0.08 \pm 0.02$ & $17.10 \pm 1.93$ & $5.99 \pm 0.23$ \\
FD & 1 & $218 \pm 3$ & $0.16 \pm 0.02$ & $16.30 \pm 1.71$ & $5.92 \pm 0.19$ \\
& 2 & $208 \pm 4$ & $0.13 \pm 0.03$ & $15.63 \pm 0.65$ & $5.42 \pm 0.12$
\end{tabular}

Table 5 Percentage suppression of P. berghei infected mice given combination treatment

\begin{tabular}{|c|c|c|c|c|c|c|}
\hline Drugs & Day 1 & Day 2 & Day 3 & Day 4 & Day 5 & Day 6 \\
\hline Nanoformulated PQ & $40.74 \pm 10.4$ & $88.72 \pm 3.3$ & $97.09 \pm 2.1$ & $88.60 \pm 5.7$ & $89.30 \pm 6.6$ & $99.44 \pm 3.3$ \\
\hline Free primaquine & $40.54 \pm 11.1$ & $68.62 \pm 2.2$ & $86.91 \pm 2.4$ & $87.16 \pm 5.3$ & $89.30 \pm 6.6$ & $90.31 \pm 7.7$ \\
\hline ED50(1:1) & $73.14 \pm 9.4$ & $95.72 \pm 9.3$ & $93.09 \pm 1.1$ & $68.60 \pm 5.7$ & $79.34 \pm 6.3$ & $89.54 \pm 3.1$ \\
\hline $\operatorname{ED} 50 / 2(1 / 2: 1 / 2)$ & $80.56 \pm 3.1$ & $78.62 \pm 5.2$ & $87.91 \pm 2.4$ & $84.06 \pm 2.3$ & $89.90 \pm 6.6$ & $91.34 \pm 5.7$ \\
\hline ED50/8(1/8 :1/8) & $86.74 \pm 1.46$ & $85.72 \pm 3.3$ & $77.09 \pm 2.1$ & $0.60 \pm 15.7$ & $-29.30 \pm 9.6$ & $-9.04 \pm 10.3$ \\
\hline Zadd(mg/kg) & $8.3 \pm 0.35$ & & & & & \\
\hline $\mathrm{Zexp}(\mathrm{mg} / \mathrm{kg})$ & $1.03 \pm 0.03$ & & & & & \\
\hline Interaction & 0.14 & & & & & \\
\hline
\end{tabular}

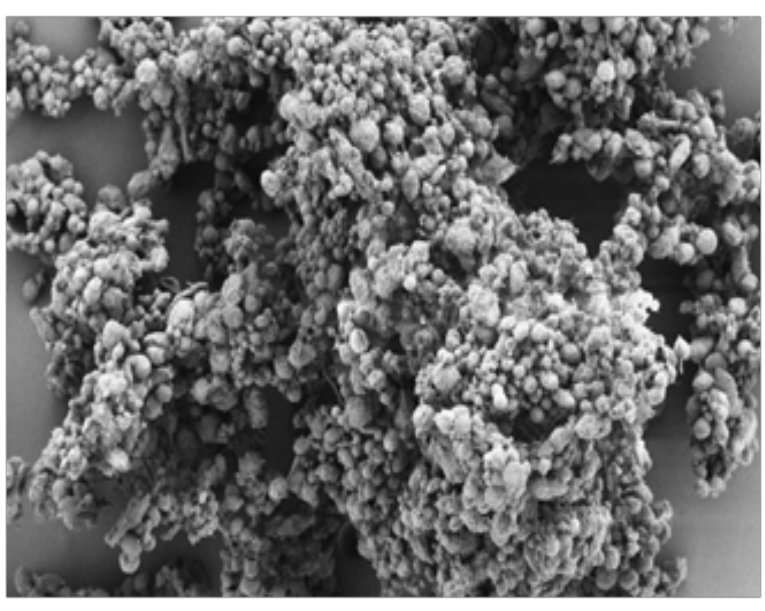

Figure I Scanning electron microscopy images of drug-loaded solid lipid nanoparticles at $\times 20,000$.

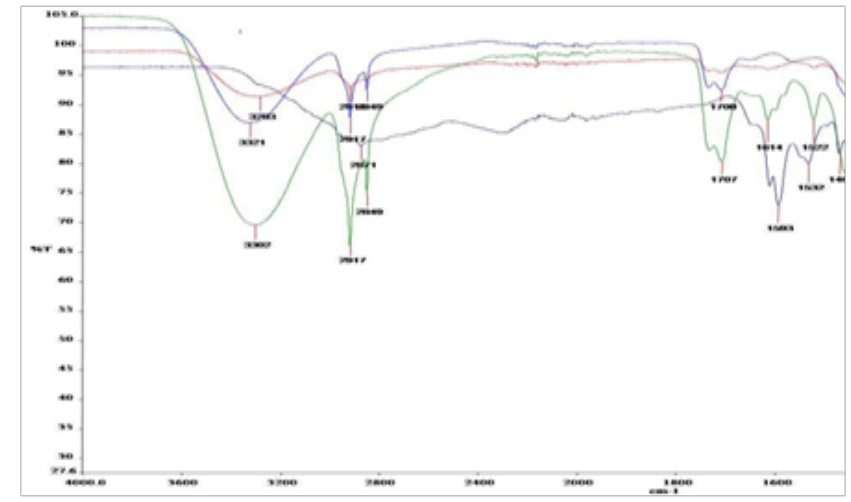

Figure 2 Fourier transform infrared spectroscopy graphs for primaquine nanoparticle formulations. First from top (A) is formulation with $5 \mathrm{ml}$ of lactose, second (B) has no drug, third (C) has $15 \mathrm{ml}$ of lactose, and the bottom one $(D)$ is free drug. 


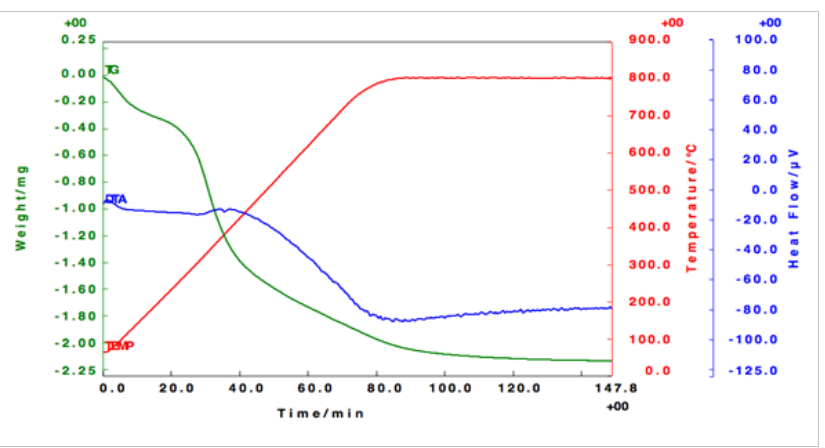

Figure 3 DTA cooling scans and heating scans. Notes: empty nanoparticles refer to solid lipid nano-particles that do not contain drug.

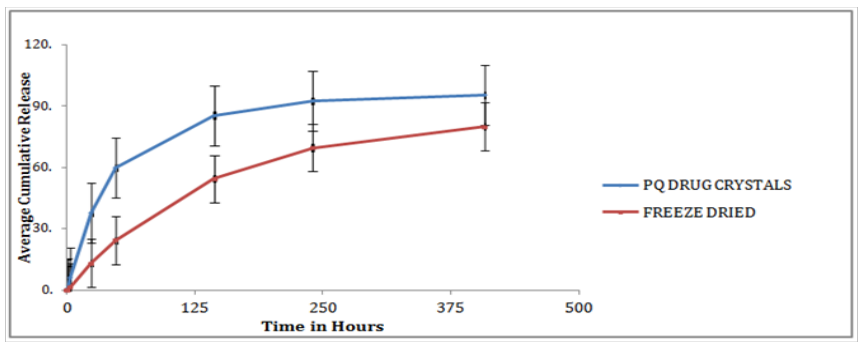

Figure 4 In-vitro release study of PQ loaded SLN in PBS pH 7.4, in $37 \mathrm{C}$ water baths. PQ Drug Crystals were used as con-trol. Data are expressed as mean $\pm S D(n=3)$. Lines drawn represent non-linear regression fit of the data.

\section{Conclusion}

PQ-SLNs were successfully formed using a modified solventemulsification evaporation method based on a w/o/w doubleemulsion. The particle size was small enough to allow directabsorption in the gastrointestinal tract. This coupled with amoderately positive surface charge is envisaged to allow forhigh absorption that could trigger an elevated concentration of PQ base in the liver. Since the drug is highly effective againsthypnozoites/liver stages of all malaria species, the formulationwill be highly efficacious, even at reduced dosages.

Aprolonged drug-release profile was observed which maypositively impact on dosage frequency and consequentlyresult in reduced toxicity. Efficacy evaluation in mice showedthat the nanoformulated PQ was $20 \%$ more effective ascompared with the conventional oral dose, indicating thegreat potential of improving antimalarial efficacy of drugsby nanoformulating them into SLNs. ${ }^{38-45}$

\section{Acknowledgements}

This study was sponsored by the Consortium for National Health Research; grant number RCDG-2012-008. National Research Foundation South Africa provided funding for our partners at CSIR. The authors sincerely thank the funding organizations for their relentless support. We also wish to express our gratitude to the CSIR, Kenya Medical Research Institute, and Institute for Primate Research for providing necessary facilities to develop the research study.

\section{Conflicts of interest}

The authors report no conflicts of interest in this work.

\section{References}

1. Jenquin MR, McGinity JW. Characterization of acrylic resin matrix films and mechanisms of drug-polymer interactions. International $J$ pharmaceutics. 1994;101(1-2):23-34.

2. Jensen DMK, Cun D, Maltesen MJ, et al. Spray drying of siRNAcontaining PLGA nanoparticles intended for inhalation. J Control Release. 2010;142(1):138-145.

3. Jeong JC, Lee J, Cho K. Effects of crystalline microstructure on drug release behavior of poly ( $\varepsilon$-caprolactone) microspheres. $J$ controlled release. 2003;92(3):249-258.

4. Karewicz A, Zasada K, Szczubiałka K, et al. "Smart" alginatehydroxypropylcellulose microbeads for controlled release of heparin. Int $J$ Pharm. 2010;385(1):163-169.

5. Leroueil-Le Verger M, Fluckiger L, Kim YI, et al. Preparation and characterization of nanoparticles containing an antihypertensive agent. European J Pharmaceutics \& Biopharmacentics. 1998;46(2):137-143.

6. Lyckfeldt $\mathrm{O}$, Rundgren DKK. Pressing and sintering developments of freeze granulated Si3N4 materials. In $27^{\text {th }}$ Annual Cocoa Beach Conference on Advanced Ceramics and Composites-B: Ceramic Engineering and Science Proceedings. 2009;24(4):331-336.

7. Mathew ST, Devi SG, Sandhya KV. Formulation and evaluation of ketorolac tromethamine-loaded albumin microspheres for potential intramuscular administration. Aaps Pharmscitech. 2007;8(1):E100-108.

8. Mello VAD, Ricci-Júnior E. Encapsulation of naproxen in nanostructured system: structural characterization and in vitro release studies. Química Nova. 2011;34(6):933-939.

9. Moriarty P. Nanostructured materials. Reports on Progress in Physics. 20001;64(3):297.

10. Moritz T, Nagy A. Preparation of super soft granules from nanosized ceramic powders by spray freezing. $J$ Nanoparticle Research. 2002;4(5):439-448.

11. Mulye NV, Turco SJ. A simple model based on first order kinetics to explain release of highly water soluble drugs from porous dicalcium phosphate dihydrate matrices. Drug development \& industrial pharmacy. 1995;21(8):943-953.

12. Negre F, Sánchez E. Advances in spray-dried pressing powder processes in tile manufacture. In Science of whitewares, USA: American Ceramic Society Ohio; 1996. p. 169-181.

13. Ohashi K, Kabasawa T, Ozeki T, et al. One-step preparation of rifampicin/ poly (lactic-co-glycolic acid) nanoparticle-containing mannitol microspheres using a four-fluid nozzle spray drier for inhalation therapy of tuberculosis. J controlled release. 2009;135(1):19-24.

14. Omwoyo WN, Ogutu B, Oloo F, et al. Preparation, characterization, and optimization of primaquine-loaded solid lipid nanoparticles. Int $J$ Nanomedicine. 2014;9:3865-3874.

15. Yan G, Li H, Zhang R, et al. Preparation and evaluation of a sustainedrelease formulation of nifedipine HPMC tablets. Drug Dev Ind Pharm. 2000;26(6):681-686. 
16. Peppas NA. Analysis of Fickian and non-Fickian drug release from polymers. Pharm Acta Helv. 1985;60(4):110-111.

17. Uchida N, Hiranami T, Tanaka S, et al. Spray-freeze-dried granules for ceramics fabrication. American Ceramic Society Bulletin. 2002;81(2):5760

18. Yokota T, Takahata Y, Katsuyama T, et al. A new technique for preparing ceramics for catalyst support exhibiting high porosity and high heat resistance. Catalysis Today. 2001;69(1-4):11-15.

19. Rundgren K, Lyckfeldt O, Sjostedt M. Improving powders with freeze granulation. Ceramic Industry. 2003;153(4):40.

20. Fontana MC, Rezer JFP, Coradini K, et al. Improved efficacy in the treatment of contact dermatitis in rats by a dermatological nanomedicine containing clobetasol propionate. Eur J Pharm Biopharm. 2011;79(2):241249.

21. Perez $\mathrm{MH}$, Zinutti $\mathrm{C}$, Lamprecht $\mathrm{A}$, et al. The preparation and evaluation of poly ( $\epsilon$-caprolactone) microparticles containing both a lipophilic and a hydrophilic drug. J Control Release. 2000;65(3):429-438.

22. Sánchez E, Moreno A, Vicent M, et al. Preparation and spray drying of $\mathrm{Al}_{2} \mathrm{O}_{3}-\mathrm{TiO}_{2}$ nanoparticle suspensions to obtain nanostructured coatings by APS. Surface \& Coatings Technology. 2010;205(4):987-992.

23. Smith G. Bioanalytical method validation: notable points in the 2009 draft EMA Guideline and differences with the 2001 FDA Guidance. Bioanalysis. 2010;2(5):929-935.

24. Master K. Spray drying handbook. 5th ed. Longman Scientific \& Technical: USA; 1991.

25. Sung JC, Padilla DJ, Garcia-Contreras L, et al. Formulation and pharmacokinetics of self-assembled rifampicin nanoparticle systems for pulmonary delivery. Pharm Res. 2009;26(8):1847-1855.

26. Takashima Y, Saito R, Nakajima A, et al. Spray-drying preparation of microparticles containing cationic PLGA nanospheres as gene carriers for avoiding aggregation of nanospheres. Int J Pharm. 2007;343(1):262-269.

27. Tanaka, N, Imai K, Okimoto K, et al. Development of novel sustainedrelease system, disintegration-controlled matrix tablet (DCMT) with solid dispersion granules of nilvadipine. J Control Release. 2005;108(2):386395.

28. Tomoda K, Ohkoshi T, Hirota K, et al. Preparation and properties of inhalable nanocomposite particles for treatment of lung cancer. Colloids Surf B Biointerfaces. 2009;71(2):177-182.

29. Varelas CG, Dixon DG, Steiner CA. Zero-order release from biphasic polymer hydrogels. J controlled release. 1995;34(3):185-192.

30. Veronese FM, Marsilio F, Caliceti P, et al. Polyorganophosphazene microspheres for drug release: polymer synthesis, microsphere preparation, in vitro and in vivo naproxen release. $J$ controlled release. 1998;52(3):227-237.
31. Wagner JG. Interpretation of percent dissolved $\square$ time plots derived from in vitro testing of conventional tablets and capsules. J Pharm Sci. 1969;58(10):1253-1257.

32. Wang S, Guo S, Cheng L. Disodium norcantharidate loaded poly ( $\varepsilon$-caprolactone) microspheres: I. Preparation and evaluation. International $J$ pharmaceutics. 2008;350(1):130-137.

33. Zhang L, Yang M, Wang Q, et al. 10-Hydroxycamptothecin loaded nanoparticles: preparation and antitumor activity in mice. $J$ controlled release. 2007;119(2):153-162.

34. Abdelwahed W, Degobert G, Fessi H. A pilot study of freeze drying of poly (epsilon-caprolactone) nanocapsules stabilized by poly (vinyl alcohol): formulation and process optimization. Int J Pharm. 2006;309(1):178-188.

35. Allen C, Maysinger D, Eisenberg A. Nano-engineering block copolymer aggregates for drug delivery. Colloids \& Surfaces B: Biointerfaces. 1999;16(1):3-27.

36. Barzegar-Jalali M, Adibkia K, Valizadeh H, et al. Kinetic analysis of drug release from nanoparticles. J Pharm Pharm Sci. 2008;11(1):167-177.

37. Barzegar-Jalali M, Alaei-Beirami M, Javadzadeh Y, et al. Comparison of physicochemical characteristics and drug release of diclofenac sodiumeudragit ${ }^{\circ}$ RS100 nanoparticles and solid dispersions. Powder Technology. 2012;219:211-216.

38. Barzegar-Jalali M, Ghanbarzadeh S, Adibkia K, et al. Development and characterization of solid dispersion of piroxicam for improvement of dissolution rate using hydrophilic carriers. BioImpacts. 2014;4(3):141148 .

39. Carriazo D, Del Arco M, Martín C, et al. Influence of the inorganic matrix nature on the sustained release of naproxen. Microporous \& Mesoporous Materials. 2010;130(1):229-238.

40. Costa P, Lobo JMS. Modeling and comparison of dissolution profiles. Eur J Pharm Sci. 2001;13(2):123-133.

41. Dubernet C. Thermoanalysis of microspheres. Thermochimica Acta. 1995;248:259-269.

42. Gibaldi M, Feldman S. Establishment of sink conditions in dissolution rate determinations. Theoretical considerations and application to nondisintegrating dosage forms. J Pharm Sci. 1967;56(10):1238-1242.

43. Higuchi T. Mechanism of sustained $\square$ action medication. Theoretical analysis of rate of release of solid drugs dispersed in solid matrices. $J$ Pharm Sci. 1963;52(12):1145-1149.

44. Hixson AW, Crowell JH. Dependence of reaction velocity upon surface and agitation. Industrial \& Engineering Chemistry. 1931;23(10):1160 1168 .

45. Javadzadeh Y, Ahadi F, Davaran S, et al. Preparation and physicochemical characterization of naproxen-PLGA nanoparticles. Colloids Surf B Biointerfaces. 2010;81(2):498-502. 